\title{
Contabilidad de Costos I, base fundamental en el área disciplinar de Contaduría Pública y Finanzas
}

\section{Cost-accounting 1, fundamental basis in the disciplinary area of Public Accounting and Finance}

Juan Carlos Benavides Fuentes ${ }^{1}$

\section{RESUMEN}

El perfil de Contaduría Pública y Finanzas, abarca cuatro áreas disciplinares: Contabilidad, Finanzas, Costos y Auditoría. Por lo tanto, es necesario poseer una base teórica y práctica en la Contabilidad de Costos I como base fundamental para las asignaturas subsecuentes del área disciplinar de Costos. En las dos primeras unidades de esta asignatura, se establecen los cimientos necesarios para comprender como funciona la contabilidad de costos y posteriormente, estos conocimientos se deben adaptar a los sistemas de costos abordados en las asignaturas subsecuentes. Para lograr este propósito, se implementaron estrategias de enseñanza y aprendizaje tales como: fabricar productos, analizar el flujo de la contabilidad de costos, utilizar un catálogo de cuentas y reflexionar con los estudiantes sobre la importancia de la asignatura, a fin de obtener el éxito académico en las siguientes asignaturas de costos, lográndose a partir de esto un mayor interés por alcanzar un aprendizaje significativo y aplicable.

Palabras claves: costos; área disciplinar; asignaturas subsecuentes.

\begin{abstract}
The Public Accounting and Finance bachelor curriculum includes four areas: accounting, finances, costing and audit. Therefore it is necessary to have a basic theory and practice in Cost-accounting 1 as a fundamental base for the subsequent subjects related to Costing. The first two units of the subject program, establish the basis needed to understand how cost-accounting works, later these knowledge should be adapted to the costing systems in the other subjects. In order to achieve this goal, teaching and learning strategies were implemented such as: create products, cost-accounting analysis, usage of counts book, and analysis with the students about the importance of the subject to reach the academic succeed in this subject, reaching more interest to have significant learning.
\end{abstract}

Keywords: costs; disciplinary area; subsequent subjects.

DOI: https://doi.org/10.5377/multiensayos.v3i6.9686

Recibido: 01 de diciembre de 2017

Aceptado: 15 de diciembre de 2017

1 Docente UNAN Managua - FAREM Estelí. Correo electrónico: juan_carlos0406@yahoo.com 


\section{INTRODUCCIÓN}

El perfil de estudio de la carrera de Contaduría Pública y Finanzas contempla cuatro áreas disciplinares: Contabilidad, Finanzas, Costos y Auditoría. En este contexto, el presente ensayo tiene como objetivo realizar una reflexión sobre la importancia de la asignatura de Contabilidad de Costos I, como base fundamental para lograr un adecuado desempeño académico en las asignaturas subsecuentes del área disciplinar de Costos, a partir de la experiencia de facilitación de esta asignatura al grupo de segundo año de la carrera de Contaduría Pública y Finanzas del turno matutino en la Facultad Regional Multidisciplinaria de Estelí.

De manera particular se pretende valorar la manera en que los contenidos del programa de la asignatura de Costos I, son esenciales para comprender los diferentes sistemas de contabilidad de costos que se abordan en las asignaturas subsecuentes, tales como el sistema de costos por procesos, el sistema de costeo ABC y el sistema de costos estándares. Es importante resaltar las sesiones de reflexión con los estudiantes a fin de concientizarlos sobre la relevancia de contar con una buena base que les permita alcanzar un aprendizaje significativo y aplicable a la realidad de las empresas en nuestro contexto. Además se expondrán las estrategias utilizadas en el proceso de enseñanza aprendizaje de esta asignatura y se analizarán los logros y dificultades que se presentaron durante la clase.

\section{DESARROLLO}

Al abordar este tópico es de suma importancia comprender el papel del contador público en diferentes entidades. Según el autor Gonzalo Sinisterra Valencia, el contador público desempeña múltiples actividades y actúa en diversos campos. Su formación profesional le permite orientar su actividad hacia la revisoría fiscal, asesoría tributaria, auditoría externa e interna, presupuestos, asesoría administrativa, diseño de sistemas de información, contabilidad de costos, etc. (Sinisterra, Contabilidad de Costos, 2006, pág. 12)

Lo planteado por este autor coincide con lo expresado en el perfil de la carrera, en el cual se especifica que el objeto de estudio de la carrera de Contaduría Pública y Finanzas abarca las áreas disciplinares de: Contabilidad, Finanzas, Costos y Auditoría, las que serán aplicadas en el control y registros de transacciones financieras en empresas públicas y privadas, para la toma de decisiones (Universidad Nacional Autónoma de Nicaragua, Managua, 2012).

La importancia de esta área disciplinar dentro de la carrera se puede resumir en los siguientes datos extraídos de la malla curricular:

- Está compuesta por las asignaturas de Contabilidad de Costos I, Contabilidad de Costos II, Contabilidad de Costos III y Contabilidad de Costos agropecuarios.

- Contempla 4 asignaturas, un total de 255 horas y 17 créditos. 
Continuando con lo expresado en el perfil de la carrera, se especifica que el área disciplinar de la contabilidad de costo, es una fase amplificada de la contabilidad general o financiera de una entidad industrial o mercantil, que proporciona rápidamente a la gerencia los datos relativos a los costos de producir o vender un artículo o de suministrar un servicio en particular. Además se encarga principalmente de la acumulación y del análisis de la información relevante para uso interno de los gerentes en la planeación, el control y la toma de decisiones.

Esta definición coincide con lo planteado por el autor Adolfo José Pastrana, quien afirma que la Contabilidad de Costos es un sistema de información que clasifica, asigna, acumula y controla los costos de actividades, procesos y productos, para facilitar la toma de decisiones, la planeación y el control administrativo (Pastrana, 2012).

Además de la definición general planteada, tanto en el perfil como por otros autores, es importante mencionar las principales funciones que desempeña un contador de costos, las cuales son abordadas en la asignatura de contabilidad de Costos I y que les permiten a los estudiantes una mejor comprensión de su papel como contador de costos.

Según Gonzalo Sinisterra Valencia, en el campo de la contabilidad de costos, al contador público le corresponde entre otras desarrollar las siguientes actividades: preparar, presentar y analizar informes de costos, interpretar informes de costos para la gerencia; diseñar sistemas de costos; preparar y presentar información general sobre el comportamiento de los costos de la empresa, en particular del área funcional de manufactura y el control de costos.

Según el perfil de la carrera, en cuanto al ámbito laboral el contador de costos puede desempeñar funciones como Contador de costos, cuyas principales funciones son:

- Clasificar, resumir y registrar la cuantificación monetaria de las operaciones de la entidad relativa a los costos de producción.

- Proporcionar la información para determinar el costo de ventas y poder determinar la utilidad o pérdida del período.

- Determinar el Costo de los inventarios, con miras a la presentación del balance general y el estudio de la situación financiera de la empresa.

- Suministrar información para ejercer un adecuado control administrativo y facilitar la toma de decisiones acertadas.

Conocer estas funciones, permite adquirir conciencia de la responsabilidad de un contador de costos y por lo tanto es necesario poseer una base teórica y práctica que se comienza a formar en la asignatura de contabilidad de costos I.

Se puede afirmar que la asignatura de Contabilidad de Costos I, es la base fundamental para las asignaturas subsecuentes del área disciplinar de Costos, porque de acuerdo con el programa en 
esta asignatura se realiza una introducción a los elementos fundamentales en materia de costos, los cuales son aplicables y necesarios en las demás asignaturas de contabilidad de costos.

Según el programa de asignatura, los contenidos abordados en esta materia permitirán al estudiante adquirir los fundamentos teóricos prácticos de la contabilidad de costos y sus elementos; así mismo podrán conocer e identificar un sistema de acumulación de costos y aplicarlo de acuerdo a las características de producción de las empresas.

En este orden de ideas, los principales contenidos para el desarrollo de las competencias de esta asignatura son: en la unidad I es Introducción a la Contabilidad de Costos, la evolución de los costos, la clasificación de los costos y el proceso del ciclo de costos; en unidad II es el Control, registros y análisis de los elementos del costos de producción, los cuales son la materia prima, la mano de obra directa y los costos indirectos de fabricación, en la unidad III Se estudian el Sistema de Costos por órdenes específicas, características del sistemas de costo por órdenes específicas, aplicación a las empresa y el registros de las diferentes órdenes de producción y servicios (Universidad Nacional Autónoma de Nicaragua, Managua, 2014).

Los contenidos reflejados en las dos primeras asignaturas son los cimientos necesarios para comprender como funciona la contabilidad de costos y por ende se debe adaptar estos conocimientos generales a los sistemas de contabilidad abordados en las asignaturas subsecuentes, como por ejemplo el sistema de costos por procesos, el sistema de costeo ABC y el sistema de costos estándares.

Entonces, ¿qué estrategias de enseñanza aprendizaje se utilizaron en el transcurso de la clase para lograr un aprendizaje significativo de las bases que se requieren en Contabilidad de Costos? A continuación se detallan algunas de estas estrategias:

- Fabricar diferentes productos (uno por grupo), lo cual permitió un aprendizaje significativo en cuanto a la identificación y clasificación de los elementos de costos, así como en la adecuada determinación del costo del producto. Entre los productos elaborados están: jabón natural de limpieza facial, crema natural de sábila, vino de guiñocuabo, botas de cuero, champú anticaspa - aclarante, tutu para niñas, tapetes de lana, café molido y fundas decoradas. Este tema en particular, representa un aspecto fundamental porque los tres elementos de costo estudiados en esta asignatura se emplean en las asignaturas subsecuentes del área disciplinar de Contabilidad de Costos. Como un elemento adicional, también es importante mencionar que con esta actividad se promovió la innovación y se fomentó el emprendimiento.

- Profundizar y analizar la lógica del flujo de la contabilidad de costo, el cual constituye uno de los temas más importantes porque es aplicable a todos los sistemas de contabilidad de costos que se abordarán en las otras asignaturas de Contabilidad de Costos contempladas en el plan de estudios de la carrera. 
- Utilizar un catálogo de cuentas para el registro contable de los elementos de costo, lo cual permite adquirir un conocimiento más consciente de la realidad de las empresas y que además establece las bases luego poder adaptarlo a las particularidades de los otros sistemas de costos que abordarán en las siguientes asignaturas de contabilidad de costos.

- Reflexionar con los estudiantes sobre la importancia de la asignatura de Contabilidad de Costos I, tanto para el avance de su plan de estudios, como para alcanzar el éxito académico en las otras asignaturas. A partir de estas reflexiones la mayoría de los estudiantes mostró mayor interés por alcanzar un aprendizaje significativo y aplicable. Los estudiantes, se involucraron en el proceso y proceso y realizaron preguntas sobre la aplicación a la realidad.

\section{CONCLUSIONES}

- El perfil de la carrera de Contaduría Pública y Finanzas establece, cuatro áreas disciplinares fundamentales, siendo una de estas la Contabilidad de Costos.

- La asignatura de contabilidad de costos I, establece los fundamentos teóricos y prácticos, necesarios para las asignaturas subsecuentes del área disciplinar de Costos.

- Tanto en el perfil de la carrera como en el programa de asignatura, se establece la relevancia de la asignatura de Contabilidad de Costos I, para el cumplimiento de las funciones de todo contador público.

- Las estrategias de enseñanza aprendizaje utilizas durante la facilitación de la asignatura de Contabilidad Costos I, tales como: fabricar productos, analizar la lógica del flujo de la contabilidad de costo, utilizar un catálogo de cuentas y reflexionar con los estudiantes sobre la importancia de la asignatura como base para las asignaturas subsecuentes, permitieron a los estudiantes una mejor integración y comprensión de la clase.

\section{BIBLIOGRAFÍA}

Pastrana, A. J. (2012). Contabilidad de Costos. Córdoba: El Cid Editor.

Sinisterra, G. (2006). Contabilidad de Costos. Bogotá: Ecoe Ediciones.

Universidad Nacional Autónoma de Nicaragua, Managua. (2012). Perfil de la carrera de Contaduría Pública y Finanzas. Managua.

Universidad Nacional Autónoma de Nicaragua, Managua. (2014). Programa de asignatura: Contabilidad de Costos I. Managua. 